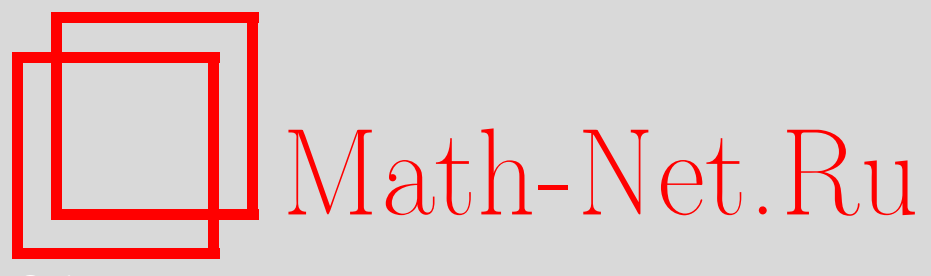

K. Ваананен, В. В. Зудилин, О линейной независимости значений рядов Чакалова, УМH, 2007, том 62, выпуск 1, 197-198

DOI: https://doi.org/10.4213/rm6119

Использование Общероссийского математического портала Math-Net.Ru подразумевает, что вы прочитали и согласны с пользовательским соглашением http://www . mathnet.ru/rus/agreement

Параметры загрузки:

IP : 54.80 .97 .219

26 апреля 2023 г., $11: 36: 21$

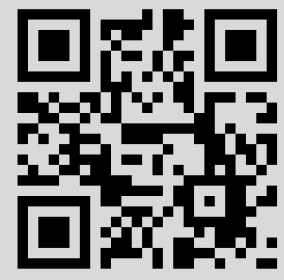




\section{О линейной независимости значений рядов Чакалова}

\section{К. Ваананен, В. В. Зудилин}

В настоящей заметке исследуются арифметические свойства значений функции Чакалова $T_{q}(z)=\sum_{\nu=0}^{\infty} q^{-\nu(\nu+1) / 2} z^{\nu},|q|>1$. Одной из открытых проблем является доказательство линейной независимости значений $T_{q}(z)$ с различными $q$; единственный результат в этом направлении [1] имеет сильные ограничения. Мы отсылаем заинтересованного читателя к обзору [2] известных результатов о линейной и алгебраической независимости значений функции Чакалова и других $q$-рядов.

Теорема 1. Пусть $t_{1}$ и $t_{2}$ - два различных положительных иелых, а числа $q \in$ $\mathbb{Z} \backslash\{0, \pm 1\}$ и $\alpha \in \mathbb{Q} \backslash\{0\}$ мультипликативно независимь ${ }^{1}$. Тогда числа $1, T_{q^{t_{1}}}(\alpha)$ и $T_{q^{t_{2}}}(\alpha)$ линейно независимы над $\mathbb{Q}$.

Отметим, что функция $f_{t}(z)=T_{q}\left(\alpha z^{t}\right), t \in \mathbb{N}$, удовлетворяет функциональному уравнению $\alpha z^{t} f_{t}(z)=f_{t}(q z)-1$. Для доказательства теоремы 1 мы по существу используем метод из [3] применительно к функциям $g_{i}(z)=f_{t_{i}}\left(z^{m_{i}}\right), i=1,2, m_{i} \in \mathbb{N}$, удовлетворяющим $\alpha_{i} z^{s_{i}} g_{i}(z)=g_{i}(q z)-q_{i}(z)$, где $q_{i}(z)=\sum_{l=0}^{m_{i}-1} \alpha^{l} q^{t_{i}}\left\{\left(\begin{array}{c}m_{i} \\ 2\end{array}\right)-\left(\begin{array}{c}m_{i}-l \\ 2\end{array}\right)\right\} z^{t_{i} m_{i} l}$, $\alpha_{i}=\alpha^{m_{i}} q^{t_{i} m_{i}\left(m_{i}-1\right) / 2}$ и $s_{i}=t_{i} m_{i}^{2}$. Масштабирование $z \mapsto z^{m_{i}}$ является новым и ключевым моментом нашей конструкции. Существует бесконечное число пар $\left(m_{1}, m_{2}\right) \in$ $\mathbb{N}^{2}$, для которых

$$
\left|s_{1}-s_{2}\right|=\left|t_{1} m_{1}^{2}-t_{2} m_{2}^{2}\right| \leqslant c_{0}
$$

с фиксированной постоянной $c_{0}>0$ (если $\tau=\sqrt{t_{1} / t_{2}}$ рационально, то мы даже можем выбрать $c_{0}=0$, в то время как в случае иррационального $\tau$ указанные пары возникают, например, из подходящих дробей непрерывной дроби для $\tau$ ). После выбора пары $\left(m_{1}, m_{2}\right)$, удовлетворяющей $(1)$, занумеруем функции $g_{1}(z)$ и $g_{2}(z)$ таким образом, что $s_{1} \leqslant s_{2} \leqslant s_{1}+c_{0}$.

С помощью принципа Дирихле строятся совместные рациональные приближения к функциям $g_{1}(z)$ и $g_{2}(z)$.

Лемма 1. Для заданных $\varepsilon, 0<\varepsilon<1 / 2$, и положительного целого $n$ существуют ненулевой многочлен $P(z)=\sum_{j=0}^{n} p_{j} z^{j} \in \mathbb{Z}[z]$ и многочлены $Q_{i}(z) \in \mathbb{Q}[z], \operatorname{deg} Q_{i} \leqslant n$, $i=1,2$, такие, что выполняются следующие условия:

(i) $\left|p_{j}\right| \leqslant|q|^{\lambda n^{2} / M+O(n)}$, где $\lambda=\lambda(\varepsilon)=(1-2 \varepsilon)(3-2 \varepsilon)^{2} /(8 \varepsilon)$ u $M=2 s_{1}$;

(ii) после домножения на $q^{n^{2} / M+O(n)}$ коэффициенты многочленов $Q_{i}(z)$ становятся иелыми;

(iii) если определить $R_{i}(z)=P(z) g_{i}(z)-Q_{i}(z), i=1,2, \operatorname{mord}_{z=0} R_{i}(z) \geqslant \frac{3}{2} n-\varepsilon n$ $u\left|R_{i}(z)\right| \leqslant|q|^{\lambda n^{2} / M+O(n)}|z|^{\frac{3}{2} n-\varepsilon n}$ при условии $|z| \ll 1$.

Применяя функциональное уравнение для $g_{i}(z)$, строим дальнейшие приближения на основе леммы 1. Именно, начиная с $R_{i 0}(z)=P_{0}(z) g_{i}(z)-Q_{i 0}(z)=P(z) g_{i}(z)-Q_{i}(z)$, $i=1,2$, определим для $k=1,2 \quad R_{i k}(z)=\alpha_{i}^{-1} z^{s_{2}-s_{i}} R_{i, k-1}(q z)=P_{k}(z) g_{i}(z)-Q_{i k}(z)$, $i=1,2$. По аналогии с [1; лемма 2] в случае $s_{1}<s_{2}$ или с [3; лемма 3] в случае $s_{1}=s_{2}$ имеет место следующая лемма о необращении в нуль.

Работа второго автора выполнена при частичной поддержке фонда INTAS, грант № 0351-5070. Начало работы было положено во время пребывания второго автора на факультете математических наук Университета Оулу в декабре 2006 г. Автор благодарит сотрудников факультета за гостеприимство.

ЭЭто означает, что единственный набор целых $i, j$, удовлетворяющих равенству $\alpha^{i} q^{j}=1$, тривиален: $i=j=0$. 
Лемма 2. Определитель

$$
\Delta(z):=\left|\begin{array}{lll}
P_{0}(z) & Q_{10}(z) & Q_{20}(z) \\
P_{1}(z) & Q_{11}(z) & Q_{21}(z) \\
P_{2}(z) & Q_{12}(z) & Q_{22}(z)
\end{array}\right| \neq 0
$$

Сравнивая степень и порядок нуля многочлена $\Delta(z)$ в нуле, заключаем, что для любого $\rho \geqslant 0$ найдется целое $\nu$ такое, что $\rho n \leqslant \nu \leqslant \rho n+2 \varepsilon n+3 s_{2}$ и $\Delta\left(q^{-\nu}\right) \neq 0$.

Зафиксируем $\varepsilon>0$ так, что $\varepsilon<\min \left\{1 / 2,1 /\left(6 c_{0}\right)\right\}$, и выберем пару $\left(m_{1}, m_{2}\right) \in \mathbb{N}^{2}$, удовлетворяющую $(1)$ и неравенству $(1+\lambda) / M<\left(1-6 c_{0} \varepsilon\right)(1-2 \varepsilon) /\left(32 c_{0}\right)$. Воспользуемся приведенной выше конструкцией с этой парой и с $\rho=\left(1-6 c_{0} \varepsilon\right) /\left(4 c_{0}\right)$. Для каждого $n$ получаем линейные формы $r_{i k}(\nu)=\alpha_{i}^{\nu} q^{\left(s_{2}-s_{i}\right) \nu(\nu+1) / 2} R_{i k}\left(q^{-\nu}\right)=$ $p_{k}(\nu) g_{i}(1)-q_{i k}(\nu), i=1,2, k=0,1,2$, причем рациональные коэффициенты $p_{k}(\nu)$ и $q_{i k}(\nu)$ после домножения на $D_{\nu} \leqslant|q|^{n^{2} / M+n \nu+O(n+\nu)}$ становятся целыми. Предполагая, что теорема 1 неверна, т.е. числа 1 и $g_{i}(1)=f_{t_{i}}(1)=T_{q^{t_{i}}}(\alpha), i=1,2$, линейно зависимы над $\mathbb{Q}$, имеем линейное соотношение $L=h_{0}+h_{1} g_{1}(1)+h_{2} g_{2}(1)=0$ с некоторыми целыми $h_{0}, h_{1}, h_{2}$, не равными одновременно нулю. Тогда

$$
0=D_{\nu} p_{k}(\nu) L=D_{\nu}\left(h_{0} p_{k}(\nu)+h_{1} q_{1 k}(\nu)+h_{2} q_{2 k}(\nu)\right)+D_{\nu}\left(h_{1} r_{1 k}(\nu)+h_{2} r_{2 k}(\nu)\right)
$$

и необращение в нуль определителя

$$
\left|\begin{array}{lll}
p_{0}(\nu) & q_{10}(\nu) & q_{20}(\nu) \\
p_{1}(\nu) & q_{11}(\nu) & q_{21}(\nu) \\
p_{2}(\nu) & q_{12}(\nu) & q_{22}(\nu)
\end{array}\right|=\left(\alpha_{1} \alpha_{2}\right)^{\nu} q^{\left(2 s_{2}-s_{1}\right) \nu(\nu+1) / 2} \Delta\left(q^{-\nu}\right)
$$

влечет существование $k \in\{0,1,2\}$ такого, что слагаемое $D_{\nu}\left(h_{0} p_{k}(\nu)+h_{1} q_{1 k}(\nu)+\right.$ $\left.h_{2} q_{2 k}(\nu)\right)$ в (2) отлично от нуля. С другой стороны, это число является целым, так что $D_{\nu}\left|h_{1} r_{1 k}(\nu)+h_{2} r_{2 k}(\nu)\right| \geqslant 1$ в соответствии с (2). Мы приходим к противоречию с тем, что $D_{\nu}\left(h_{1} r_{1 k}(\nu)+h_{2} r_{2 k}(\nu)\right) \rightarrow 0$ при $n \rightarrow \infty$ согласно лемме 1 и нашему выбору параметров. Тем самым теорема 1 доказана.

Теорема 1 остается верной при замене $\mathbb{Q}$ на произвольное мнимое квадратичное поле $\mathbb{I}$, а $\mathbb{Z}$ на кольцо $\mathbb{Z}_{\mathbb{I}}$ целых поля $\mathbb{I}$. Метод из [3] и небольшая модификация доказательства теоремы 1 приводит к следующему общему результату (ср. с [1]).

Теорема 2. Пусть $q \in \mathbb{Z}_{\mathbb{I}},|q|>1$. Предположим, что $t_{1}, \ldots, t_{l}-$ различные положительные челые и ненулевое число $\alpha \in \mathbb{I}$ мультипликативно независимо с $q$. Тогда $l+1$ чисел 1 и $T_{q^{t} k}(\alpha), k=1, \ldots, l$, линейно независимы над II. Кроме того, если q и $\alpha_{1}, \ldots, \alpha_{l}$ из $\mathbb{I} \backslash\{0\}$ мультипликативно независимы, то числа 1 и $T_{q^{t}}\left(\alpha_{k}\right)$, $k=1, \ldots, l$, линейно независимы над $\mathbb{I}$.

В доказательстве этой теоремы условие (1) заменяется неравенством, вытекающим из теоремы Кронекера о совместных приближениях.

Подчеркнем, что ввиду количественного характера метода, применяемого в наших доказательствах, можно оценить снизу линейные формы с целыми коэффициентами от рассматриваемых чисел.

\section{Список литературы}

[1] M. Amou, K. Väänänen, Monatsh. Math., 144:1 (2005), 1-11. [2] P. Bundschuh, Sūrikaisekikenkyūsho Kōkyūroku, 1219 (2001), 110-121. [3] K. Väänänen, Math. Ann., 325:1 (2003), $123-136$.

\section{K. Ваананен (K. Väänänen)}

Department of Mathematical Sciences,

University of Oulu, Finland

E-mail: kvaanane@sun3.oulu.fi

\section{В. В. Зудилин (W. Zudilin)}

Московский государственный университет

им. М. В. Ломоносова;

Математический институт им. В. А. Стеклова РАН

E-mail: wadim@mi.ras.ru
Представлено А. Г. Сергеевым Принято редколлегией 18.01.2007 